\title{
ON THE DISTRIBUTION OF OZONE IN THE DIRECT CURRENT CORONA
}

\author{
BY ERIC K. RIDEAL AND JAKOB KUNZ
}

The mechanism by which ozone is formed from oxygen in the various diverse methods of preparation such as chemical, photochemical, thermochemical, or by collision with the aid of $\alpha$-particles or electrons and finally by the silent electric discharge ${ }^{1}$ the most general method, is by no means clearly understood. It is generally assumed that the reaction proceeds in stages as follows:

$$
\begin{aligned}
& \mathrm{O}_{2} \rightleftarrows \mathrm{O}+\mathrm{O} \\
& \mathrm{O}_{2}+\mathrm{O} \rightleftarrows \mathrm{O}_{3}
\end{aligned}
$$

a somewhat more plausible hypothesis than the assumption of a termolecular reaction of the type

$$
{ }_{3} \mathrm{O}_{2} \rightleftarrows{ }_{2} \mathrm{O}_{3}
$$

It might be suggested, however, that the mechanism of ozone formation proceeds through a series of dimolecular reactions of the type

$$
\begin{aligned}
& \mathrm{O}_{2}+\mathrm{O}_{2} \rightleftarrows \mathrm{O}_{4} \\
& \mathrm{O}_{4}+\mathrm{O}_{2} \rightleftarrows \mathrm{O}_{3}
\end{aligned}
$$

All these hypotheses assume that ozone is not produced through the intermediary of gas ions or electrons, except insofar as the ions or electrons serve to activate the oxygen molecule. The hypothesis that ozone can be produced without gas ionization was indicated by Lenard ${ }^{2}$ and supported by Ludlam. ${ }^{3}$ In all reactions, however, in which ozone is produced the presence of gas ions can be detected, and the possibilities of ozone formation by the interaction of gas ions are much more varied than in the preparation of $\mathrm{H}_{3}$ owing to the relative complexity of the oxygen molecule. The experiments of Sir J. J. Thom-

${ }^{1}$ For a résumé of the literature on the formation of ozone see $F$. O. Anderegg: Jour. Am. Chem. Soc., 39, 258 I (1917) and forthcoming book "Ozone" by one of the authors.

2 Ann. Phys., (4) I, 480 (I 900 ).

3 Phil. Mag., 23, 757 (1912). 
son $^{1}$ and $\mathrm{F}$. Horton ${ }^{2}$ have indicated the existence of electric allotropes of oxygen of the form $\ddot{O}, \mathrm{O}^{\prime} \mathrm{O}^{\prime}, \mathrm{O}^{\prime \prime}$, as well as positively charged polymers of electric molecular weights $\left(\frac{a \mathrm{M}}{n e}\right.$, as $a$ is an integer, $M$ the atomic weight, and ne the electron charge), $8,16,32,48,96$. We can thus also assume the formation of ozone through ionic reactions of the types

$$
\begin{aligned}
& \mathrm{O}_{2} \longrightarrow \dot{\mathrm{O}}+\mathrm{O}^{\prime} \\
& \dot{\mathrm{O}}_{2}+\mathrm{O}^{\prime}=\mathrm{O}_{3} \\
& \mathrm{O}_{2}{ }^{\prime}+\dot{\mathrm{O}}=\mathrm{O}_{3}
\end{aligned}
$$

These, however, presuppose not only an activation of the molecule but a subsequent ionization in addition, entailing a greater expenditure of energy.

Chemically, there is little evidence for the existence of atomic oxygen, not only on account of the great reactivity which this element would possess but also from a study of the decomposition of ozone. The decomposition of ozone, if a monomolecular reaction, would proceed through a series of reactions as in ( $\mathbf{I}$ ) while if bimolecular according to (2) or (3). In the free state the decomposition of ozone is bimolecu$1 \mathrm{ar}^{3}$ except in the presence of solid catalysts ${ }^{4}$ where a monomolecular reaction ensues owing to the possibility of removal of the third oxygen atom without its

$$
\mathrm{O}_{3}+2 \mathrm{Ag}=\mathrm{O}_{2}+\mathrm{Ag}_{2} \mathrm{O}
$$

temporary existence in the free state.

$$
\mathrm{O}_{3}+\mathrm{Ag}_{2} \mathrm{O}=2 \mathrm{Ag}+{ }_{2} \mathrm{O}_{2}
$$

The heat of formation of ozone which is endothermic is approximately 34,000 calories per $\mathrm{g}$ mol. ${ }^{5}$ An approximation to the heat of formation of ozone may also be made on the

'Phil. Mag., 13, 5or (1907), et seq. *

2 Ibid., 22, 2I4 I9I4).

${ }^{3}$ Chapman and Jones: Jour. Chem. Soc., 93, 1638 (1908); 97,2463 (1910); Weigert, Zeit, phys. Chem., 80, 78 (1912).

${ }^{4}$ J. Strutt: Proc. Roy. Soc., 87, 302 (1913).

5 Jahn: Zeit. anorg. Chem., 60, 357 (1908); 68, 250 (1910). 
radiation theory of chemical action. ${ }^{1}$ With Perrin ${ }^{2}$ we may make no assumption as to the mode of mechanism but write the equation of reaction

$$
{ }_{3} \mathrm{O}_{2}={ }_{2} \mathrm{O}_{3}
$$

and assume that the wave length of the ozonizing light is I65 $\mu \mu$ (the mean of Warburg's ${ }^{3}$ and Regener's values ${ }^{4}$ who noted that the absorption band extended from I $20 \mu \mu$ to $I 80 \mu \mu$ ) and that of the deozonizing light $260 \mu \mu$. (The mean of the deozonizing absorption band limits 230 to $290 \mu \mu$.) From the relationship

$$
Q=\mathrm{N} h r_{\mathrm{O}_{2}}-\mathrm{N} h r_{\mathrm{O}_{3}}=\mathrm{N} h\left(\mathrm{I} .8 \times \mathrm{IO}^{15}-\mathrm{I} . \mathrm{I} 5 \times \mathrm{IO}^{15}\right)
$$

where $\mathrm{N}$ is the Loschmidt number $=6.062 \times \mathrm{Io}^{23}$ and $h$ Planck's constant $6.585 \times 10^{-27}$, we obtain the value $Q=$ 62,830 cals or 31,415 cals per $g$ mol. of ozone. (Perrin's $3 \mathrm{I}, 000$ with approximate values of $\mathrm{N}$ and $h$.)

This method of calculation, however, although leading to a satisfactory numerical result, is open to serious objection from the standpoint of the quantum hypothesis, for it will be noted that Perrin has arbitrarily assumed the values of $\mathrm{N} h r$ derived from an assumed maximum line in a broad band to correspond to one g. mol. of reactant, oxygen, and one $\mathrm{g}$ mol. of product, ozone, arriving at the heat of formation of two $\mathrm{g}$ mols of ozone. It is tacitly assumed that the ozonizing light falls on a molecular complex of 3 molecules of oxygen acting as one, and the deozonizing light quantum is emitted from a complex consisting of two molecules of ozone. Now we have seen that in homogeneous decomposition the mechanism is bimolecular and, therefore, this latter assumption, viz., that ozone only decomposes when two molecules come in contact with one another and that photochemical decomposition accelerates the reaction only by increasing the number of effec-

1 Haber: Ber. deutsch. phys. Ges., I3, III $(191$ I); W. McC. Lewis: Jour. Chem. Soc., III, 1086 (I917); Perrin: Ann. de Physique, II, 5 (1919).

2 Loc. cit.

${ }^{3}$ Deutsch. phys. Ges. Verh., I7, io (1915'.

4 Ann. Phys., (4) 20, 1033 (rgo6). 
tive contacts (virtually a photochemical decomposition of $\left.\left(\mathrm{O}_{3}\right)_{2}\right)$ might be accepted although it is more reasonable to suppose that one quantum of energy is derived from each molecule. The formation of ozone, however, through termolecular contacts which enduce the complex with properties so as to make it act as an individuum and capable of being activated by a single large quantum seems highly improbable.

W. McC. Lewis ${ }^{1}$ adopts the hypothesis that ozone may be formed by the activation of each of the oxygen molecules to an equal degree, calculating from Chapman and Jones' figures ${ }^{1}$ on the thermal decomposition of ozone, the value of $\mathrm{Nhr}_{\mathrm{O}_{3}}=$ Io,69o cals, is obtained. Adopting the expression $3 \mathrm{O}_{2}=2 \mathrm{O}_{3}$ to explain the mechanism of ozone formation and the heat of formation as 69,000 cals per $2 \mathrm{~g}$. mol. we obtain with Lewis a value for the critical energy increment for each oxygen molecule per g. mol. Nhr$r_{\mathrm{O}_{2}}=30,127 \mathrm{cal}$ corresponding to $\lambda=904 \mu \mu$. Thus it should be possible to produce ozone from oxygen by illumination with light near the visible red end of the spectrum. It is, however, unknown whether photochemical ozone formation does take place at this point, and as will be readily observed, this photochemical synthesis involves the simultaneous coincidence of, not only three oxygen molecules, but of three activated oxygen molecules which would result in an extremely small reaction velocity.

It, therefore, appears likely that ozone can be photochemically prepared by at least two distinct methods, by illumination with light of wave length $\lambda=$ Iooo $\mu \mu$ and by illumination with light of wave length $120-180 \mu \mu$, the former being represented by the usual reaction $3 \mathrm{O}_{2}=2 \mathrm{O}_{3}$, while for the latter we must adopt some other hypothesis than the simultaneous coincidence of three activated oxygen molecules.

The energy of activation of oxygen to its highest point may be calculated in a variety of ways, thus the ionizing potential of oxygen is found at 9.2 volts. From the relationship

${ }^{1}$ Loc. cit. 
$h r=\mathrm{V} e$ and $Q_{2}=\mathrm{N} h r$, we obtain the value $Q_{\mathrm{O}_{2}}=2 \mathrm{IO}, 68 \mathrm{o}$ calories. Nernst ${ }^{1}$ from e. m. f. measurements showed that the equilibrium concentrations of ozone and the active form of oxygen if it existed would be in the ratio of $10^{20}: \mathrm{r}$. Or, the heat of formation on the assumption that atomic oxygen and ozone were the reactants would be approximately in the ratios $\frac{20}{3}:$ I. Since $Q_{O_{s}}=34,000$ calories per g. mol., $\mathrm{Q}_{\mathrm{O}_{2}}=226,900$ calories per g. mol. Again, we have already noted that the ozonizing band lies within the spectral region $120-180 \mu \mu$, and it is reasonable to make the assumption that activation can take place by irradiation with a band or more probably a series of lines and not only with a single line, owing to the fact that some molecules in the gas are already partly activated with infra-red quanta (see ante). These partly activated molecules, of course, require less energy, or light of longer wave length, than those not activated at all or which have received no critical energy increment from thermal radiation. Adopting $\mathbf{x} 20 \mu \mu$ as the wave length necessary to activate a non-active oxygen molecule we obtain the value

$$
N h r_{\mathrm{O}_{2}}=233,300 \text { cals }
$$

The mean value obtained from these three determinations is $\mathrm{Nhr}_{\mathrm{O}_{z}}=223,620$ calories per g. mol. or I I I,8Io calories per g. atom of oxygen Nhro (since one quantum splits up a molecule forming two atoms). It is uncertain in what form the active oxygen exists but since the value of $Q$ exceeds by far the dissociation value of hydrogen $\mathrm{H}_{2}=\mathrm{H}+\mathrm{H}$ - Ioo,ooo cals per g. mol. and the computed value for nitrogen $Q=$ 160,000 to 190,000 cals per g. mol. it would appear likely that this value for the activation of oxygen includes not only the dissociation into atoms $\mathrm{O}_{2}=\mathrm{O}+\mathrm{O}$, but also the secondary ionization of those atoms $\mathrm{O}=\dot{\mathrm{O}}+\Theta$; the liberated electron then attaching itself to a neutral oxygen molecule, $\ominus+$ $\mathrm{O}_{2}=\mathrm{O}_{2}^{\prime}$, and a subsequent reaction produces ozone $\mathrm{O}_{2}^{\prime}+$

${ }^{1}$ Zeit. Elektrochemie, 9, 891 (1903). 
$\mathrm{O}=\mathrm{O}_{3}$, a hypothesis supported by the value derived from the ionizing potential of oxygen.

For the activation of the oxygen molecule but a small quantum of energy is required. McC. Lewis has calculated , a value of ${\mathrm{N} h \mathrm{O}_{2}}_{2}=30, \mathrm{I} 27$ calories from Chapman and Jones' figures $^{1}$ for such an activation, while Ladenberg and Lehman ${ }^{2}$ have noticed lines in the red and infra red position of the oxygen ozone spectrum. A distinct line is said to exist at $\lambda=$ I040 $\mu \mu$, corresponding to a value of $\mathrm{Nhr}_{\mathrm{O}_{2}}=28$,000 cals, or a mean value of $\mathrm{Nhr}_{\mathrm{O}_{2}}=29,060$ calories per'g mol.

For the photochemical decomposition of ozone taking the value in the region $230-290 \mu \mu$ adopted by Perrin, viz., $260 \mu \mu$ the critical energy increment for ozone is $\mathrm{Nhr}_{\mathrm{O}_{3}}=$ ro9,00o cal per $\mathrm{g}$ mol. Thus, the heat of formation of ozone is given by the relationship

$$
\begin{aligned}
Q & =\mathrm{N} h r_{\mathrm{O}}+\mathrm{N} h r_{\mathrm{O}_{2}}-\mathrm{N} h r_{\mathrm{O}_{3}} \\
& =\text { II }, 810+29,060-109,000=3 \mathrm{r}, 870 \mathrm{cal} \text {. per g. mol. }
\end{aligned}
$$

By the silent discharge in oxygen $\mathrm{I} 8 \mathrm{o} \mathrm{g}$ per $\mathrm{kw} \mathrm{hr}$. is the best yield obtained; according to the above calculation where I40,870 calories are required per $\mathrm{g}$ mol., the theoretical yield should be $295 \mathrm{~g}$ per $\mathrm{kw}$ hr., or a technical efficiency of 61 percent obtains in practice. It may be noted if $r_{O}$ corresponding to a value of $\lambda=\mathrm{I} 20 \mu \mu$ represents the frequency necessary to split up a molecule into its atoms and also remove an electron from the atom the corresponding photochemical decomposition band $\lambda=230-290 \mu \mu$ should contain the light of the correct frequency not only to decompose ozone but to ionize it as well. It is hoped to investigate this point experimentally at a later date, but it appeared necessary first to determine the distribution of ozone in the corona discharge taking place in oxygen, and further, to note whether the light usually apparent in the corona tube was accompanied by any ultraviolet light as would be expected on the assumption that the formation of ozone is accompanied by the emission of light within the wave lengths $230-290 \mu \mu$.

${ }^{1}$ Loc. cit,

2 Ber. deutsch. chem. Ges., 4, 175 (1906). 
Experimental.-The corona tube consisted of a thin brass tube $25.4 \mathrm{cms}$ long and $2.54 \mathrm{cms}$ internal radius, along the axis of which was stretched a bright platinum wire $0.4 \mathrm{I} \mathrm{mm}$ in diameter. The ends of the tube were sealed with thin pieces of quartz glass and gas inflow and eflow tubes were provided at each end. The corona was excited by means of a series of direct current coupled dynamos driven at a constant speed by means of a voltage regulator. The average voltage as determined by a calibrated Kelvin electrometer was 16,500 volts and no difficulty was obtained in maintaining this value when the wire was the seat of positive discharge and the tube earthed and negative (positive corona). With a negative corona on the other hand, fluctuations in voltage ( \pm 250 volts) were frequent.

A slow stream of dry oxygen was passed through the tube at a steady rate of to liters per hour, and the distribution of ozone in the oxygen between the wire and the tube was determined by two independent methods.

(a) Chemically.-A fine glass capillary was inserted at right angles to the axis and in the middle of the tube. Through the capillary, the tip of which could be adjusted to various positions in the annular space between the wire and the tube wall, a sample of the gas could be withdrawn and analyzed by aspiration through a solution of $2 N$ potassium iodide. The rate of aspiration was I liter per hour. All glass connections were employed and the analysis was performed by titration, after acidification, with sodium thiosulphate solution ( $10 \mathrm{cc}=$ I $\mathrm{mg}$ ozone) which was standardized before and after each set of determinations.

(b) Photochemical Analy'sis.-In order to confirm the chemical analysis, and provide against distortion of the field in the corona tube by the insertion of any dielectric a photo analytic method was devised. For this purpose the method of Krüger and Möller $^{1}$ and of Fabry and Buisson" was modified so as to determine directly the distribution of ozone in the corona at

\footnotetext{
${ }^{1}$ Phys. Zeit., 13, 779 (1912).

${ }^{2}$ Comptes rendus, 156,789 (1913).
} 
different distances from the wire. A diagram of the general arrangement is given in Fig. I.

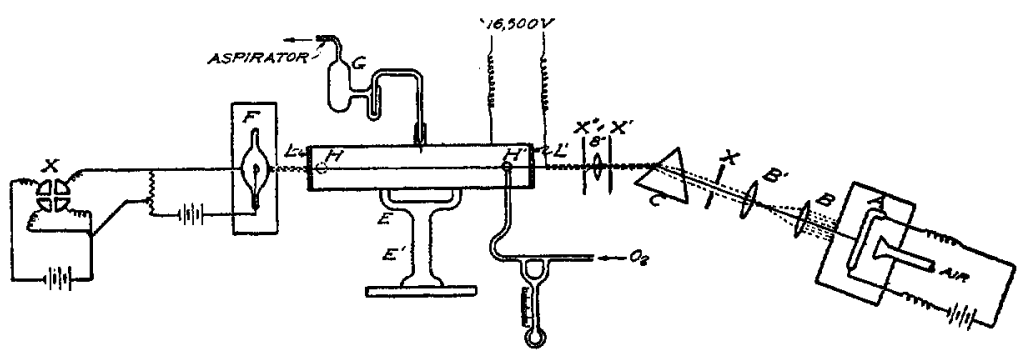

Fig. I

A parallel beam of ultraviolet light (ca $2 \mathrm{~mm}$ wide and broad and within the spectral region $\lambda=250$ to $35^{\circ} \mu \mu$, obtained, through the slits $\mathrm{XX}^{\prime} \mathrm{X}^{\prime \prime}$, from a quartz mercury vapor lamp $A$ by the quartz optical system of lenses $B^{\prime} B^{\prime \prime}$ and prism $C$, was passed through the quartz plates $I^{\prime} I$, of the corona tube $\mathrm{E}$ provided with the gas inlet and outlet tubes $\mathrm{H}^{\prime} \mathrm{H}$, and gas sampling arrangement $\mathrm{G}$. The distance of the glass capillary of the sampling tube and of the ultraviolet light beam from the platinum wire in the corona tube could be adjusted by means of the screw thread $\mathrm{E}^{\prime}$.

Much trouble was caused by the alteration in intensity of the ultraviolet light from the lamp with the time, this was finally minimized but not entirely removed by keeping the lamp cool and at a uniform temperature by means of an air blast. The intensity of the emergent beam was measured by means of a photoelectric cell $\mathrm{F}$. This photoelectric cell constructed of clear quartz and containing potassium was made extremely sensitive by submitting the alkali metal to the action of a spark discharge in hydrogen before replacement of this gas by helium. The applied voltage to the potassium cell was 163 volts and the alteration in current measured in the usual way by means of a sensitive electrometer shunted across a high resistance in the main circuit.

Owing to the fact that the corona was excited by means 
of a high $d$. c. voltage of 16,500 volts, elaborate precautions has to be taken to ensure against electrical leakage to the photoelectric cell circuit. This leakage was finally reduced so that the deflection of the galvanometer produced by the corona was less than I mm.

The concentration of ozone in the corona tube was calculated on the basis of de Beer's law $I_{\text {emergent }}=I_{\text {entrant }} e^{-k c d}$ where $I_{\text {emergent }}$ and $I_{\text {entrant }}$ are the intensities of the emergent and entrant beams, the deflection of the electrometer being assumed strictly proportional to the intensity of the light falling on the photoelectric cell; $d=$ the thickness of the gas column $25.4 \mathrm{~cm}, c$ the concentration of ozone in percent by volume, and $k$ the specific absorption coefficient or extinction coefficient of ozone. Owing to the fact that the dispersion obtained by the optical system was rather small and that a relatively wide beam of ultraviolet light was passed through the corona tube, no definite value could be assigned to $K$, such as those given by Meyer ${ }^{1}$ or Krüger and Möller. ${ }^{2}$ The mean $\mathrm{K}$ value was accordingly calculated from a comparison of the figures obtained in this way with the determinations by the chemical method and found (approximately.) equal to 2.30.

The results obtained by the two methods are indicated in the following curves, where the ordinates represent percentage of ozone by volume in the oxygen and the abscissae the distance in millimeters from the wire or tube axis to the point where the determination was made (Fig. 2). As examples of the figures obtained may be cited the following:

Chemical Method.-Positive corona.

Distance from wire, o mm.

Strength of the thiosulphate, ro cc. $=1.3728 \mathrm{mg}$. $\mathrm{O}_{3}$.

Titre for $500 \mathrm{cc}$. (aspirated at one liter per hour), I7, $17.3,17.2,17.4 \mathrm{cc}$ thiosulphate solution.

Mean percent by volume, 0.220 .

Optical Method.-Negative Corona.

${ }^{1}$ Ann. Phys., (4) I7, 849 (1903).

2 Loc. cit. 
Owing to the fact that the corona light affected the potassium photoelectric cell, it was found necessary to perform the operations in the following manner: After having obtained the zero of the instrument the ultraviolet light intensity was measured after passing through the tube containing oxygen.

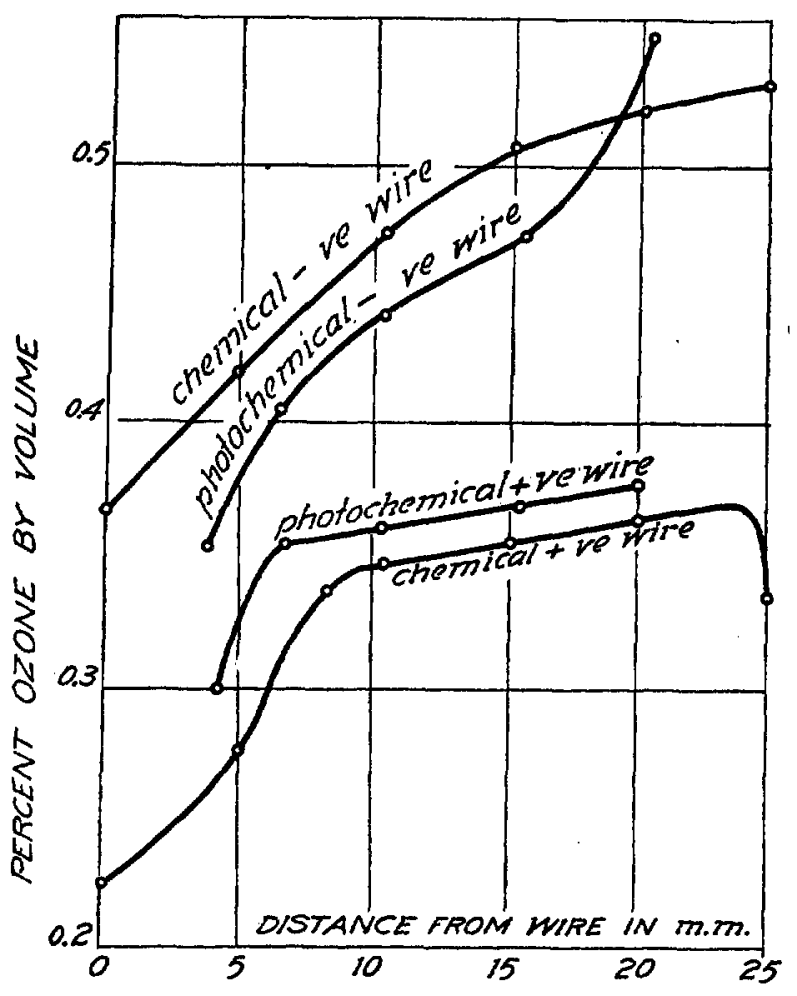

Fig. 2

The corona was then turned on and the new deflection obtained, somewhat graater than before owing to the effect of the additional light derived from the corona. The discharge was allowed to pass until a stationary deflection of the galvanometer was obtained, a period of from three to five minutes, being usually, amply sufficient, the reading thus obtained being noted, the corona discharge was then stopped and the 
new deflection immediately determined as instanced by the following figures:

Applied voltage, I6,500 volts.

Current, o.68 milliampere.

Distance from wire, $15.5 \mathrm{~mm}$. to center of beam.

Deflection of electrometer with ultraviolet light cut off $=$ deflection $50 \mathrm{~mm}$.

Deflection of electrometer with oxygen in tube, 190.2 mm. (mean of 3 ).

Deflection of electrometer with oxygen in tube when corona light appears $194 \mathrm{~mm}$. (mean of three).

Mean deflection (I) without corona light, $140.2 \mathrm{~mm}$; (2) with corona light, $144 \mathrm{~mm}$.

Deflectior of electrometer when dynamic equilibrium has been established with corona light, I61.5 mm. (mean of three); after corona is cut off, $156 \mathrm{~mm}$. (mean of three); mean deflection with corona light, III.5 mm.; without corona light, $106.5 \mathrm{~mm}$.

Inserting these values in the equation

$$
\log _{e} \frac{\mathrm{I}}{\mathrm{I}_{0}}=-k c d
$$

where $d=25.4 \mathrm{~cm}$. and $\mathrm{K} .=2.30$, we obtain values for $c=0.462$ percent, 0.469 percent, average 0.465 percent.

It will be noticed that the distribution of ozone in the corona varies very notably with the sign of the discharge, the negative corona producing higher concentrations than the positive, this in agreement with Warburg's results. ${ }^{1}$ According to the data obtained by Anderegg ${ }^{2}$ although in a closed corona tube the total quantity of ozone formed is practically identical for a positive or negative corona yet it will be observed that the rate of formation of ozone as deduced from the slope of the $c, t$ curves obtained by him is considerably greater for a negative corona, and one would, therefore, infer that in a corona tube through which oxygen was passing continuously

\footnotetext{
1 Loc. cit.

${ }^{2}$ Jour. Am. Chem. Soc., 39, 259 I (1917).
} 
the yield from a negative corona would exceed that from a positive, which is actually found to be the case.

The average yield of ozone obtained was as follows: Negative corona, $0.045 \mathrm{~g}$ per coulomb; positive corona, 0.024 $\mathrm{g}$ per coulomb. While Warburg obtained with a negative corona $0.043 \mathrm{~g}$ per coulomb, and with a positive $0.017 \mathrm{~g}$ per coulomb.

It has usually been assumed (see I) that ozone formation occurs in the corona, or in the luminous part of the discharge; in the case of the positive corona it will be noted that there is a rapid rise in ozone concentration until a distance of $7 \mathrm{~mm}$ from the wire is reached after which the ozone concentrated remains approximately constant. This rapid rise can be accounted for on the assumption either that the ozone is photochemically destroyed in the neighborhood of the corona, or that the ozone molecules lose electrons and as a consequence the positive ozone gas ions are repelled from the wire and thus give rise to the phenomenon of corona pressure. In this case it is evident that the ozone molecules must lose electrons more easily than oxygen or that the ionizing potential of ozone must be appreciably lower than oxygen (see ante).

In the case of the negative corona, a somewhat different type of curve is obtained and it would appear that in this case the ozone is again made at the positive electrode, as in the case of the positive corona, and diffuses from the positive electrode, the tube, to the wire where a slight decomposition obtains. It does not seem possible to account for the steady rise of the ozone concentration away from the wire on the assumption that ozone is formed in the luminous part of the negative corona.

The corona discharge is accompanied not only by the emission of visible light but of ultraviolet light in addition. Comparative figures for the fraction of ultraviolet light (between $\lambda=200 \mu \mu$ and $\lambda=400 \mu \mu)$ emitted from the positive and negative corona were obtained by measuring the intensity of radiation falling on the photoelectric cell through the quartz plate (lower limit $\lambda=200$ ) and noting the fall in radia- 
tion intensity by the insertion of a thin glass plate (lower limit of transmission $\lambda=400 \mu \mu$ ). The following figures were obtained:

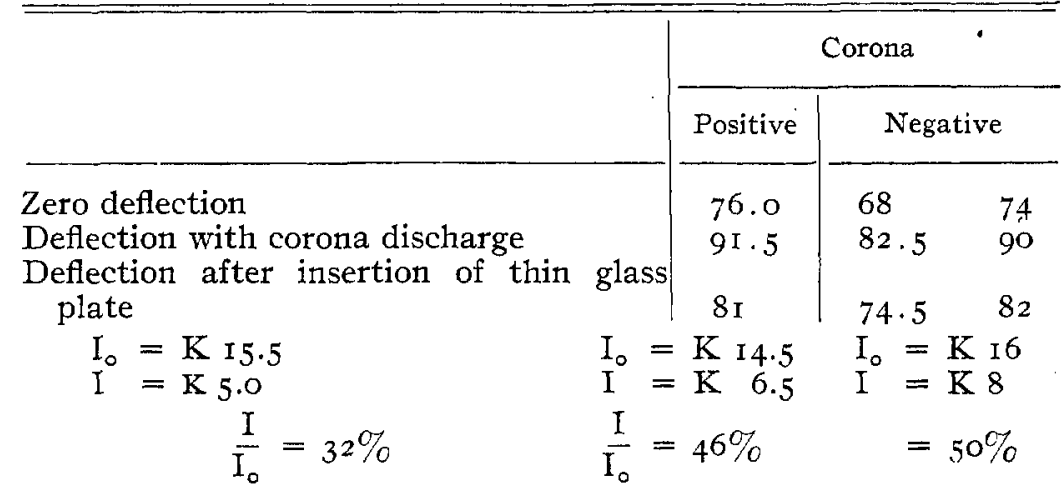

Indicating that only 32 percent of the radiation received by the photoelectric cell in the case of the positive corona lies within the region $200-400 \mu \mu$ and 68 percent in the ultraviolet portion of the spectrum, while with the negative corona the percentage of visible light emitted is nearly 50 percent. The actual ratio of ultraviolet light to visible in the corona is probably much higher than indicated by the figures owing to the partial absorption of the same when passing through the column of oxygen gas, since no light of wave length below $200 \mu \mu$ is capable of passing through. The fact that the ratio of ultraviolet to visible light is less in the negative corona than that in the positive, although the ozone production is higher, substantiates the view that the ozone formation in the negative corona is not caused by radiation at the wire surface.

\section{Summary}

The various methods by which ozone can be prepared in the light of the radiation hypothesis are reviewed, and the conclusion is drawn that molecular species of one kind can be activated by radiation to different extents.

The distribution of ozone in the direct current corona has been measured by two independent methods; by chemical 
analysis and photometrically with the aid of potassium photoelectric cell of quartz. It is shown that the distribution of ozone in the positive direct current corona differ in a marked manner from that obtaining in the negative corona.

The ultraviolet light emission from the corona exceeds that of the visible and in the corona itself the ratio of ultraviolet to visible radiation is probably exceedingly high.

The writers are indebted to Professor A. P. Carman for the use of the dynamos in the corona laboratory.

University of Illinois

March 8, 1920 